\title{
Combinatorial Properties of Basic Encryption Operations
}

\author{
Extended Abstract
}

\author{
Thilo Zieschang \\ ARCOR \\ KöIner Strasse 12, 65760 Eschborn, Germany \\ email: zieschang@acm.org
}

\begin{abstract}
The basic ingredients of modem fast software block encryption schemes are computer instructions like SHIFT, ADD, XOR etc. We analyze the algebraic structure of different combinations of those cryptographic primitives from a purely combinatorial point of view. Different subsets of such operations will yield an interesting variety of different permutation groups, e.g. semidirect products, affine linear groups, wreath products, and symmetric groups. As we will show, a simple pair of a SHIFT and an ADD operation is already powerful enough to generate every possible encryption function on its set of input blocks. On the other hand, any possible combination of SHIFT and XOR operations can only produce a subset of at most $n 2^{n}$ functions within the symmetric group of order $n !$. The present results are useful in theory at first. Their cryptographic applications can be found in providing practical tools for the analysis of the algebraic structure of new block encryption schemes and evaluation of their subroutines.
\end{abstract}

\section{Introduction}

One of the main goals in secret key cryptography is the development of design criteria for good block ciphers. Several necessary conditions are known which have to be fulfilled by every secure cipher. To support fast software encryption, many of the recently developed block ciphers are compositions exclusively of efficient, basic computer instructions like SHIFT, XOR, and ADD, for example. Further, arithmetic operations like exponentiation or multiplication in Fermat prime moduli have been used. Examples of such encryption schemes are IDEA, SAFER, RC5 and others. A lot of trial and error is involved in the development of new block ciphers. Different subsets and combinations of the above basic operations result in completely different levels of security. Besides such properties as confusion, diffusion, avalanche, nonlinearity, resistance against several known cryptanalytic attacks, etc., it is an important criterion that a given encryption algorithm realizes a large variety of different permutations among its binary input vectors. The importance of those combinatorial properties on the security of block ciphers had been pointed out by several authors. Nevertheless, few systematic work has been done in this area so far. Thus, it is important to analyze the combinatorial structure of those basic cryptographic functions which are the underlying components of most encryption schemes. We will study different combinations of basic operations with respect to the above security measure. By analyzing their group generating properties we answer the question how many different encryption functions can be realized by given subsets of those operations, and which is their corresponding cycle structure. Another important question in terms of resistance against cryptanalytic 
attacks is whether the generated permutation group is a primitive or an imprimitive group. Suppose we have a pair $(P, C)$ of plaintext and ciphertext, $C=E_{k}(P)$, and assume that the encryption algorithm $E$ generates an imprimitive group $G$. Let $\Delta_{\mathrm{i}}$ and $\Delta_{\mathrm{j}}$ be blocks for $G$ with $P \in \Delta_{i}$ and $C \in \Delta_{j}$. Then, according to the definition of imprimitivity, every other plaintext $\mathrm{P}^{\prime}$ which is also contained in $\Delta_{i}$ will automatically be mapped into $\Delta_{\mathrm{j}}$. Since this can considerably reduce the search amount, this property would severely undermine the security of an encryption scheme. Thus it is necessary to verify whether a given block cipher generates an imprimitive or a primitive group.

It is the intention of this present work to provide useful results and proofs that can be employed in the analysis of combinatorial properties of block ciphers. Note that combinatorial results like those below are designed to simplify the determination of the specific group generated by a given algorithm. In practice, however, we have to make use of additional randomness assumptions to consider the data-dependence of our encryption algorithm appropriately.

As one can see, a rich algebraic structure is involved when combining different sets of computer instructions. Among others, the following groups of permutations do occur: symmetric groups of different degree, semilinear products, wreath products, and affine general linear groups.

\section{Basic Operations}

First, we have to identify a set of simple instructions that are bijective functions on the set of n-bit binary vectors. Subsequently, we will refer to such functions as basic operations. Consider the addition of two variables, for example. If we add always some fixed value $c$, we get a function ADD_c, which operates on single input variables. All basic operations can be regarded as permutations, which allows us in the present context to speak about their cycle structure, element order, and so on. Since we study CPU registers, their length $\mathbf{n}$ to be used below will often be a power of two.

It is an interesting question whether it is feasible to realize every possible permutation as a product of simple basic operations? As we will see - the answer is yes. This is even possible if we restrict ourselves to products consisting of only two different, fixed basic operations.

\subsection{Elementary Properties of Individual Basic Operations}

In this section we will introduce some of the individual basic operations which have to be studied in more detail.

XOR_c This function operating on binary vectors of length $\mathrm{n}$ works as follows. The input value is XOR'ed with some arbitrary, but constant value $c$. If $\mathrm{c}$ is not equal to the all-zero string, then we get an involution. One of the transpositions, say (a,b), in the cycle decomposition of XOR_c can be constructed as desired, by choosing an appropriate $c$ value. We can simply take $c=(a$ XOR $b)$, so that a maps on $b$. By choice of $2^{n}-1$ different fixed values $c$, this yields $2^{n}-1$ different fixed point-free involutions. 
Note that XOR_c is always an even permutation, since the number of transpositions in its cycle decomposition is equal to $2^{n-1}$.

Example: let $n=4$ and suppose that $c=[1010]$. We identify the set of 4-bit strings with the corresponding decimal numbers $0,1, \ldots, 15$. This yields the following permutation: $X O R \_c \equiv(0,10)(1,11)(2,8)(3,9)(4,14)(5,15)(6,12)(7,13)$.

SHIFT_k We will understand this function as a logical shift (rotation) to the right by $k$ positions. The order of this permutation is equal to $n / \operatorname{gcd}(k, n)$. Further, the permutation is not fixed point-free and contains cycles of different lengths dividing $n$. Shifting by a different number of positions gives n-1 nontrivial permutations of this type. We can determine the fixed points of SHIFT_k explicitly. Let the variable X represent an arbitrary bit string of some fixed length. If $\mathrm{X}=101$, for example, then XXXX stands for a string of the form 101101101101. The function SHIFT_k has exactly $2^{\text {gcd(k,n) }}$ fixed points of form $X X . X$, where $X$ is an arbitrary string of length $\operatorname{gcd}(k, n)$.

It is easy to determine the cycle structure of a SHIFT_k permutation. Since SHIFT_k = $(\text { SHIFT_1 })^{\mathrm{k}}$, it is sufficient to examine SHIFT_1. Let $\left[\mathrm{a}_{1}, \mathrm{a}_{2}, \ldots, \mathrm{a}_{\mathrm{n}}\right]=\left[\mathrm{X}_{1}, \mathrm{X}_{2}, \ldots, \mathrm{X}_{\mathrm{b}}\right]$ be $\mathrm{a}$ partition of the input vector into $b$ subblocks of equal length $m, b m=n$, such that those subblocks do not allow further subdivision of the form $X_{i}=Y Y$, where $Y$ is a subblock of length $m / 2$. Then, in the cycle decomposition of SHIFT 1 , the element $\left[a_{1}, a_{2}, \ldots, a_{n}\right]$ is contained in a cycle of length $\mathrm{m}$. This implies the following numbers of cycles. The number of elements contained in $n$-cycles corresponds with the number of $n$-bit vectors $\left[X_{1}, X_{2}\right]$ with $X_{1} \neq X_{2}$. This is equal to $2^{n} \cdot 2^{n / 2}=\left(2^{n / 2}-1\right) 2^{n / 2}$. Hence, SHIFT_1 possesses $\left(2^{n / 2}-1\right) 2^{n / 2} / n$ cycles of length $n$. In general, the number of elements contained in $n / m-$ cycles, $m \mid n$ and $m<n$, corresponds with the number of $n$-bit vectors $\left[\left(X_{1} X_{2}\right)^{m}\right]$ with $X_{1}$ $\neq X_{2}$. Hence, SHIFT_l contains $\left(2^{n / m}-2^{n / m}\right) \mathrm{m} / \mathrm{n}$ cycles of length $\mathrm{n} / \mathrm{m}$. As we can see from the above, for every given $b$, the number of cycles of length $b$ is a multiple of two, which shows that SHIFT_1, and hence SHIFT_k $=(\text { SHIFT_1 })^{\mathrm{k}}$, is always an even permutation.

Example: let $n=4$. Again, we identify the set of 4-bit strings with the corresponding decimal numbers $0,1, \ldots, 15$. For $\mathrm{k}=1$, for example, we get the permutation SHIFT_1 $\equiv$ $(0)(1,2,4,8)(3,6,12,9)(5,10)(7,14,13,11)(15)$.

ADD_c This function represents addition modulo $2^{n}$ of the constant value $c$, ignoring overflow. $A D D_{-} 1$, for example, results in the $2^{\mathrm{n}}$-cycle $\left(0,1,2, \ldots, 2^{\mathrm{n}}-1\right)$. $A D D \_, 0<c<2^{n}$, is always a fixed point-free, regular permutation, which means that it consists of $k$ cycles of length $m, m \neq 1$, such that $\mathrm{km}=n$. The cycle length $m$ is equal to $\mathrm{m}=2^{\mathrm{n}} / \mathrm{gcd}\left(\mathrm{c}, 2^{\mathrm{n}}\right)$. This shows that ADD_c is not always an even permutation. ADD_c is an odd permutation if and only if the resulting cycle decomposition yields a $2^{n}$-cycle, which is the case for those $c$ with $\operatorname{gcd}\left(c, 2^{m}\right)=1$. Hence, ADD_c is even if and only if $c$ is even.

Example: let $\mathrm{n}=4$ and suppose that $\mathrm{c}=5$. This yields the permutation $\mathrm{ADD}_{-} 5 \equiv(0,5$, $10,15,4,9,14,3,8,13,2,7,12,1,6,11)$.

MUL_c This operation means multiplication modulo $2^{\text {n }}$ (ignoring overflow) by some constant $c$, where $c$ is odd and $0<c<2^{n}$. MUL_c is a bijective mapping, if and 
only if, $\operatorname{gcd}\left(\mathrm{c}, 2^{\mathrm{n}}\right)=1$, which yields the above condition on possible values for $\mathrm{c}$. Zero and $2^{n-1}$ are always fixed points; odd numbers are always mapped on odd, and even numbers are mapped on even numbers.

Example: let $\mathrm{n}=4$ and $\mathrm{c}=5$, then we get the function MUL_ 5 with the following cycle structure: MUL_ $5 \cong(0)(1,5,9,13)(2,10)(3,15,11,7)(4)(6,14)(8)(12)$.

The length of every cycle in the permutation MUL_c divides $2^{n-2}$, since $\left(Z / 2^{n} Z\right)^{*} \cong$ $(\mathrm{Z} / 2 \mathrm{Z}) \times\left(\mathrm{Z} / 2^{\mathrm{n}-\mathrm{Z}} \mathrm{Z}\right)$. MUL_c produces odd permutations as well as even permutations, depending on $\mathrm{c}$; more exactly, the following holds.

Lemma: MUL_c is an even permutation even if and only if $\mathrm{c} \equiv 1 \bmod 4$.

Proof: Every element $a \in\left(Z / 2^{n} \mathrm{Z}\right)^{\circ}$ has one of two possible forms: either $a=4 m-1$ or a $=4 m^{\prime}+1$, with $m, m^{\prime} \in N$. In both cases, the square $a^{2}$ of a has the same form, $a^{2}=4 k+$ 1 , where $k=(4 m-2) m$ or $k=\left(4 m^{\prime}+2\right) m^{\prime}$, respectively. Hence, if $b \in\left(Z / 2^{n} Z\right)^{*}$ is a quadratic residue, then it follows that $b \equiv 1 \bmod 4$. Since $\left(Z / 2^{n} Z\right)^{*}$ has exactly $2^{n-2}$ quadratic residues, as well as it has exactly $2^{\mathrm{n}-2}$ elements $b$ with $b \equiv 1 \bmod 4$, and since further every quadratic residue necessarily yields an even permutation, this already proves the assertion.

MULT_c For values $\mathrm{c}$ such that $\operatorname{gcd}\left(\mathrm{c}, 2^{\mathrm{n}}+1\right)=1$, MULT_c can be understood as the (bijective) function which multiplies the input variable by the constant $\mathrm{c}$, modulo $2^{n}+1$. To see how this works, we give a short example. While computing with our binary strings, we will not identify them with the set of numbers $\left\{0,1, \ldots, 2^{n}-1\right\}$. Instead we will take the set $\left\{1,2, \ldots, 2^{n}\right\}$, thus replacing zero by $2^{n}$. If $2^{n}+1$ is prime, then MULT_c is a fixed point-free, regular permutation. This can be seen as follows. If MULT_c were not regular, then an appropriate power of MULT $\mathrm{c}$ would have some fixed points. This, however, is impossible, since nonzero multiplication in a prime modulus is invertible, which implies that $c a \neq a$ for every $c \neq 1$ and $a \neq 0$. The operation MULT_c can efficiently be executed by a method called "low-high multiplication“", as described in Lai [Lai], page 35 .

Lemma: The basic operation MULT_c, considered as a permutation on the set of $n$-bit binary vectors, is even if and only if $\mathrm{c}$ is a quadratic residue modulo $2^{n}+1$.

Proof: The set $\left\{\right.$ MULT_c $\left.\mid 1 \leq c \leq 2^{n}\right\}$ forms a group $G$ of order $2^{n}$ containing both, odd and even permutations. This follows, since $G$ always contains an odd cycle MULT_d of length $2^{\mathrm{n}}$, where $d$ is a generator of the multiplicative group of the prime field $\mathrm{Z} /\left(2^{\mathrm{n}}+1\right) \mathrm{Z}$. Each permutation group containing odd elements has an equal number of odd and even elements.

If $\mathrm{c}$ is a quadratic residue modulo $2^{\mathrm{n}}+1$, then MULT_c must be an even permutation. But $\left(Z /\left(2^{n}+1\right) Z\right)^{*}$ has exactly $2^{n-3}$ quadratic residues, hence each quadratic nonresidue e $\in\left(Z /\left(2^{n}+1\right) Z\right)^{*}$ must yield an odd permutation MULT_e. This proves the assertion.

Example: let $\mathrm{n}=2$, then $2^{\mathrm{n}}+1=5$ is prime; as a result, the following permutations do occur: MULT_2 $\cong(1,2,4,3)$, MULT_3 $\cong(1,3,4,2)$, MULT_4 $\cong(1,4)(2,3)$. 


\subsection{Closure and Generating Properties of Individual Functions}

We have the following generator properties of individual basic operations. Here, $<\mathrm{M}>$ denotes the group generated by the set $M$.

$\left\langle\left\{X O R_{-} c \mid c \in\{0,1\}^{n}\right\}\right\rangle=\left\langle\left\{X O R_{-} c_{j} \mid 1 \leq j \leq n\right\}\right\rangle$, where $c_{j}:=\left[b_{1} b_{2} \ldots b_{n}\right]$, with $b_{i}=\delta_{i j}$. The resulting group is an elementary abelian 2-group of order $2^{n}$, which is isomorphic to $\left(Z_{2}\right)^{n}$. Every minimal generating set for this group comprises of $n$ elements.

$<\left\{S_{H I F T} \mathrm{k}|0 \leq \mathrm{k}\langle\mathrm{n}\}\rangle=\langle\right.$ SHIFT_1 $1>$. The generated group is isomorphic to the cyclic group of order $n$.

$\left\langle\left\{A_{D} \_c \mid 0 \leq c<2^{n}\right\}\right\rangle=\langle$ ADD_1 $1>$. The generated group is isomorphic to the cyclic group of order $2^{\text {n. }}$

$\left\langle\right.$ MULT_c $\left.\mid 0 \leq \mathrm{c}\left\langle 2^{\mathrm{n}}\right\}\right\rangle$, where $\mathrm{p}:=2^{\mathrm{n}}+1$ is prime. Then the multiplicative group $\left(Z_{p}\right)^{*}$ is cyclic and we find a generating element g such that $\langle$ MULT_c $\left.\left.| 0 \leq c<2^{n}\right\}\right\rangle=$ $<$ MULT_g $>$. Hence, this group is isomorphic to a cyclic group of order $2^{n}$. If $2^{n}+1$ is not prime, then the structure of the multiplicative group of $\mathrm{Z} /\left(2^{\mathrm{n}}+1\right) \mathrm{Z}$ varies in accordance with $\mathrm{n}$. In practice, however, we use the operation MULT only in Fermat prime moduli, which is the case for $n=8$ and $n=16$, for example.

$<\left\{\right.$ MUL_c $\mid \mathrm{c}$ odd, $\left.0 \leq \mathrm{c}\left\langle 2^{\mathrm{n}}\right\}\right\rangle=\langle$ MUL_a, MUL_- 1$\rangle$, where $\mathrm{a}$ is an element of order $2^{n-2}$. The multiplicative group of $\mathrm{Z} /\left(2^{\mathrm{n}} \mathrm{Z}\right)$ is an abelian group of order $2^{\mathrm{n}-1}$. This group is isomorphic to $\mathrm{Z} /\left(2^{\mathrm{n}-2} \mathrm{Z}\right) \times \mathrm{Z} /(2 \mathrm{Z})$.

\section{Combination of Basic Operations}

In the above sections we have studied several types of basic operations that can be used in the construction of fast software encryption schemes. Now the question arises which specific subsets of those basic operations should be combined together to achieve a large number of encryption functions, i.e. permutations of binary n-bit vectors, with presumably high structural complexity? Therefore, we have to analyze the group generating properties of different combinations of those basic operations. In the present paper, due to its space limitations, we restricted ourselves to a collection of some interesting pairs of generators, resulting in different permutation groups.

As we will see, there are surprisingly big differences among slightly different mixtures. For example, consider two different cryptosystems operating on binary input vectors of length $\mathrm{n}$, the first consisting of a combination of SHIFT and XOR operations, while the second system is using a combination of SHIFT and ADD operations. Then the first scheme will only be able to produce at most $\mathrm{n}^{\mathrm{n}}$ different, simple-structured "encryptions", while the second scheme has the power to generate the whole amount of $2^{\text {n! }}$ possible encryption functions.

If we further mention the result that, taken two arbitrary permutations from the symmetric group of degree $n$, it is most probable that those two elements already 
generate the whole alternating or symmetric group (probability $\rightarrow 1$ for $n \rightarrow \infty$ ), then it is even more surprising that the groups generated by different pairs of basic operations are much smaller in most cases.

\section{$3.1\{X O R$, SHIFT\} : Semidirect Products}

Theorem: The group $G$ generated by the set $S:=\left\{S H I F T \_d, X O R \_c \mid 0 \leq d<n, c \in\right.$ $\left.\{0,1\}^{n}\right\}$ of all SHIFT and XOR operations is isomorphic to a semidirect product $G=N U$ of an elementary abelian 2-group $\mathrm{N}$ of order $2^{n}$ by a cyclic group $\mathrm{U}$ of order $\mathrm{n}$. The order of $\mathrm{G}$ is equal to $\mathrm{n} 2^{\mathrm{n}}$ and $\mathrm{G}$ can be generated by $n+1$ elements. If $n=2^{k}$ is a power of 2 , then $\mathrm{G}$ is a nonabelian 2-group of order $2^{\mathrm{k}+\mathrm{n}}$. The maximal required word length with generating set $S$ is equal to 2 .

Proof: Suppose we have a finite sequence $a_{1} a_{2} \ldots a_{m}$ of XOR and SHIFT operations. Then it is always possible to replace this sequence by a single XOR operation, followed by a single SHIFT operation (or, alternatively, first SHIFT and afterwards XOR). The reason for this is the following obvious exchange property:

XOR_e $\cdot$ SHIFT_d =SHIFT_d $\cdot X O R \_c$, where $c:=S H I F T \_-d(e)$.

Hence, we only have to enumerate all $n 2^{n}$ possible words SHIFT_d $\cdot$ XOR_c to get all elements of the group generated by the set $\left\{\right.$ SHIFT_d, XOR_c $\left.\mid 0 \leq d<n, \bar{c} \in\{0,1\}^{n}\right\}$. The cardinality of this group is equal to $n 2^{n}$ : Suppose that SHIFT_d $d_{1} \cdot X O R \_c_{1}=$ SHIFT_ $d_{2} \cdot X O R \_c_{2}$. Then left multiplication of both sides by SHIFT_- $d_{1}$ yields: XOR_c $c_{1}=S H I F T \_\left(d_{2}-d_{1}\right) \cdot X O R \_c_{2}$. If we apply both sides to the register $\bar{A}:=c_{2}$, then we get $\left(c_{1}\right.$ XOR $\left.c_{2}\right)=0$. It follows that $c_{1}=c_{2}$, which further implies that $d_{1}=d_{2}$. We have shown that the group generated by XOR and SHIFT operations is of order $n 2^{n}$.

Next we have to determine the structure of this group. The set $N:=\left\{X O R \_c \mid c \in\right.$ $\left.\{0,1\}^{n}\right\}$ of XOR operations is a subgroup of the alternating group of degree $2^{n}$ and is isomorphic to an elementary abelian 2 -group, $N \cong\left(Z_{2}\right)^{n}$. The set $U:=\{$ SHIFT_d $\mid 0 \leq d$ $\langle n\}$ is isomorphic to a cyclic subgroup of order $n$. Further, let XOR_c $\in N$ and SHIFT_d $\in \mathrm{U}$ be two arbitrary elements of the above sets, then


$\mathrm{e}:=\mathrm{SHIFT}-\mathrm{d}(\mathrm{c})$. Hence, $\mathrm{N}$ is a normal subgroup of the product group $\mathrm{UN}^{-}=\mathrm{NU}$. Since $N \cap U=\{\overline{i d}\}$, this shows that the set $\left\{\right.$ SHIFT_d, XOR_c $\left.\mid 0 \leq d<n, c \in\{0,1\}^{n}\right\}=N U$ is a semidirect product of $\mathrm{N}$ by $\mathrm{U}$ whose order is $\mathrm{n} 2^{n}$.

If the register length $n=2^{k}$ is a power of two, the order of $G$ is also a power of two and $\mathrm{G}$ is a nonabelian 2-group of order $2^{\mathrm{k}+\mathrm{n}}$. $\mathrm{G}$ can be generated by $\mathrm{n}+1$ elements: one SHIFT and $\mathrm{n}$ XOR operations, as shown in the previous section. If we take the generating set $\left\{\right.$ SHIFT_d, XOR_c $\left.\mid 0 \leq d<n, c \in\{0,1\}^{\mathrm{n}}\right\}$ for $\mathrm{G}$, then the diameter of the corresponding Cayley graph is equal to 2 . 


\section{2 [ADD, SHIFT\} : Symmetric Groups of Degree $2^{n}$}

Theorem: The group $G$ of degree $2^{n}, n \in N$, generated by the set $\left\{A D D \_c, S H I F T\right.$ d $\mid 0$ $\left.\leq \mathrm{c}<2^{\mathrm{n}}, 0 \leq \mathrm{d}<\mathrm{n}\right\}$ of all ADD and SHIFT operations is isomorphic to the symmetric group of degree $2^{\text {n }}$.

Proof: Our strategy is to construct a specific group element $g \in G$ whose existence within primitive groups of sufficiently large degree forces the group to be isomorphic to the alternating or symmetric group of corresponding degree. Therefore, our first task is to prove that the generated group $\mathrm{G}$ is not imprimitive. Suppose that $\mathrm{G}$ is imprimitive and $\Delta \subset \Omega:=\left\{0,1,2, \ldots, 2^{n+1}\right\}$ is a nontrivial block for $G$ (i.e. $1<|\Delta|<2^{n}$ ) containing zero, $0 \in \Delta$. If $\Delta$ contains an odd element $a, 0<a<2^{n}$, then $a=A D D \_a(0)$ and therefore $\Delta=A_{D D} \mathrm{a}(\Delta)$. Further, this implies that $\Delta=\left(\right.$ ADD_a $^{\mathrm{i}}(\Delta)$ for every $0 \leq \mathrm{i}<\mathrm{n}$. But for odd a we have $\left\langle\left\{\left(A D D_{-} a\right)^{i}|0 \leq i\langle n\}\rangle=\left\langle A D D_{-} 1\right\rangle\right.\right.$. Hence, it follows that $\Omega=$ $\angle A D D_{1} 1>(0) \subseteq \Delta$, which shows that in this case $\Delta=\Omega$, a contradiction. Hence, $\Delta$ contains only even numbers. Let $k \in \Delta$ be such an even number, $k \neq 0$. Then the binary $n$-bit register representing the integer $k$ contains at least one bit equal to 1 . By an appropriate power SHIFT_c of SHIFT_1, this , $1^{\text {" }}$ can be shifted by c positions to the least significant bit of our register, the result of this operation thus representing an odd number. Since 0 is a fixed point of SHIFT_c, we see that SHIFT_c $(\Delta)=\Delta$. But then we also have SHIFT_c(k) $\in \Delta$, which is a contradiction, since, by construction, SHIFT_c(k) is odd. Hence, the group $\mathrm{G}$ must be primitive.

The second step of our proof is the construction of an appropriate group element $g$, from which we can deduce that $\mathrm{G}$ contains the alternating group of degree $2^{n}$. Here, we will show that the primitive group $G$ of degree $2^{n}$ always contains a $2^{\text {n-1 }}$-cycle $g$. Let $s$ be a cycle, $s=\left(s_{1}, s_{2}, s_{3}, \ldots, s_{r}\right)$, and let $t$ be an arbitrary permutation. Then one can verify that it always holds the equation $t^{-1}=\left(t\left(s_{1}\right), t\left(s_{2}\right), t\left(s_{3}\right), \ldots, t\left(s_{r}\right)\right)$; the conjugate of $s$ has the same cycle structure than $s$ itself. It is obvious that the same then holds for arbitrary permutations $\mathrm{s}$, $\mathrm{s}$ consisting of two or more nontrivial cycles.

Now consider the $2^{n}$-cycle ADD_1 $=\left(0,1,2,3, \ldots, 2^{n}-1\right) \in G$. By the above observation we see that $\mathrm{a}:=$ SHIFT_ $_{1} \cdot \mathrm{ADD}_{-} 1 \cdot \mathrm{SHIFT}_{-}-1=$ $=($ SHIFT_1(0), SHIFT_1(1), SHIFT_1(2), SHIFT_1(3), ..., SHIFT_1(2n - 1)).

For $0 \leq \bar{m} \leq 2^{n-1}-1$ we have SHIFT_ $1(m)=2 \mathrm{~m}$, since the rightmost bit in the binary representation of $m$ is zero. Hence, in this case the logical shift SHIFT_1 is equivalent to an arithmetic shift. For $2^{n-1} \leq m \leq 2^{n}-1$ we can write $m=2^{n-1}+m^{\prime}, \overline{0} \leq m^{\prime} \leq 2^{n-1}-1$. This allows us to see that for such $m$ we get SHIFT_1(m) $=$ SHIFT_1 $\left(2^{\mathrm{n}-1}\right)+$ SHIFT_ $1\left(\mathrm{~m}^{\prime}\right)=1+2 \mathrm{~m}^{\prime}$. Therefore, the above permutation is equal to $\mathrm{a}=(0,2,4$, $\left.6, \ldots, 2^{n}-2,1,3,5, \ldots, 2^{n}-1\right)$. From this, by setting $e:=2^{n-1}$, we get $a^{c}=(0,1)(2,3)(4$, 5)...( $\left.2^{n}-2,2^{n}-1\right)$. Finally, we compute $g:=A D D_{-}-1 \cdot a^{e}=\left(2^{n}-1,2^{n}-3,2^{n}-5, \ldots, 3,1\right)$. This element $g$ fixes every even number and, as desired, is a cycle of length $2^{n-1}$. Since every primitive permutation group of degree $2^{n}$ containing a cycle of length $2^{n-1}$ is (at least) $\left(2^{\text {n-1 }}+1\right)$-fold transitive ([Wiel], Theorem 13.8), we see that for register length $n, n$ $>2$, the group $\mathrm{G}$ must be 9 -fold transitive. But (see the classification of finite simple groups and [Came], for example) we know that in this case $\mathrm{G}$ must be isomorphic to either the alternating or symmetric group of corresponding degree. Since G contains odd 
elements (take the $2^{n}$-cycle ADD_1, for example), $\mathrm{G}$ is isomorphic to the symmetric group of degree $2^{n}$. Also, for $n=2$, we get $G=\left\langle A D A_{-}\right.$, SHIFT_ 1$\rangle=\langle(0,1,2,3),(1,2)\rangle$ $\cong \mathrm{S}_{4}$.

\section{3 [MUL, SHIFT : Symmetric Groups of Degree $2^{n}-1$}

Since 0 is a fixed point for MUL as well as for SHIFT operations, it is reasonable to consider zero as an exceptional value here. Therefore, we will subsequently consider the operation of $\mathrm{G}=\langle$ MUL_c, SHIFT_d $| 0<\mathrm{c}<2^{\mathrm{n}}$, $\mathrm{c}$ odd, $\left.0 \leq \mathrm{d}<\mathrm{n}\right\}>$ on the set of register variables $\Omega^{c}=\Omega-\{0\}=\left\{1, \overline{2}, 3, \ldots, 2^{n}-1\right\}$.

Theorem: The group $\mathrm{G}=\left\langle\left\{\right.\right.$ MUL_c, SHIFT_d $\mid 0<\mathrm{c}<2^{\mathrm{n}}$, $\mathrm{c}$ odd, $\left.0 \leq \mathrm{d}<\mathrm{n}\right\}>$ of degree $2^{\mathrm{n}}-1, \mathrm{n} \in \mathrm{N}$, generated by all MUL and SHIFT operations acting on the set $\Omega^{\mathrm{b}}=$ $\Omega-\{0\}$ of order $2^{n}-1$, is isomorphic to the symmetric group of degree $2^{n}-1$.

Proof: Our strategy is as follows. First we show that $\mathrm{G}$ is transitive, primitive, and then, by a quite technical procedure, we construct an element in $G$ which is a 3-cycle.

Let $x, y \in \Omega^{\prime}$ be two arbitrary points. First we show that there exists a permutation $\pi \in$ $\mathrm{G}$ such that $\pi(\mathrm{x})=\mathrm{y}$.

Case 1: $x$ and $y$ are odd. By MUL $y$, since $y$ is odd, we can map 1 onto $y$. In the same way we can map $x$ onto 1 by taking the inverse of MUL_ $x$, i.e. multiplication by $x^{-1}$. Combining both operations yields MUL_ $x^{-1} y(x)=y$, as desired.

Case 2: $x$ even, $y$ odd. Since $x \neq 0$, we can shift $x$ by an appropriate number $k$ of positions, such that the least significant bit of $x^{\prime}:=$ SHIFT_k(x) is equal to , 1 “, thus $x^{\prime}$ is odd. Now we can apply case (1) above on $x^{\prime}$ and map $x^{\prime}$ onto $y$. This shows that a combination of one SHIFT followed by one MUL operation can map $\mathrm{x}$ onto $\mathrm{y}$.

Case 3: $x$ odd, $y$ even. By simply taking inverse permutations, this is the same as in (2).

Case 4: $x$ and $y$ are even. This is an immediate consequence of cases (2) and (3).

Hence, the group $\mathrm{G}$ acts transitive on $\Omega^{6}$. However, we note that, in the general case, we need a sequence of not only two but three basic operations to map two arbitrary even numbers onto each other. The resulting operation has the form SHIFT - MUL - SHIFT.

Now we have to show that the above transitive operation is primitive. Let $\Delta$ be a block for $G$ which contains -1 . If we assume $|\Delta|>1$, then $\Delta$ contains an element $\mathrm{c}$ having zeros and ones in its binary representation. Since SHIFT $1(\Delta)=\Delta$, this shows that $\Delta$ contains both, even as well as odd elements: simply shift $\mathrm{c}$ by an appropriate number of positions.

Consider the permutation MUL_ $\left(2^{n-1}+1\right) \in$ G. MUL $\left(2^{0-1}+1\right)$ is an involution that fixes every even element and consists of transpositions of the form $\left(x, x+2^{n-1} \bmod 2^{n}\right)$. This can be seen as follows. Let $x=2 y+1$ be odd. Then MUL $\left(2^{n-1}+1\right)(x)=\left(2^{n-1}+\right.$ $1)(2 y+1)=2^{n} y+2^{n-1}+2 y+1=x+2^{n-1}\left(\bmod 2^{n}\right)$. Further, $\left(2^{n-1}+1\right)^{2}=1\left(\bmod 2^{n}\right)$, which shows that the order of MUL $\left(2^{n-1}+1\right)$ is equal to two. As we have noted, $\Delta$ contains even elements, and therefore MUL_ $\left(2^{n-1}+1\right)(\Delta)=\Delta$. It follows that, for every odd element $\left[b_{n-1}, b_{n-2}, \ldots, b_{1}, 1\right]$ in $\Delta$, we also have $\left[1-b_{n-1}, b_{n-2}, \ldots, b_{1}, 1\right] \in \Delta$.

Suppose we have a number in $\Delta$ whose binary representation has the form $\left[\ldots, b_{i+2}, 1, b_{i}\right.$, ....], then we can shift the „1" to the rightmost position, replace $b_{i}$ by $1-b_{i}$, shift back 
and finally get the element $\left[\ldots, b_{i+2}, 1,1-b_{i}, \ldots.\right] \in \Delta$. Thus, we can construct every binary $n$-bit vector which is different from the all-zero vector by starting from $-1=[1,1$, $\ldots, 1]$ and then replacing 1 's and 0 's accordingly. This is always possible if we start constructing each substring of adjacent zero positions by beginning replacement with the rightmost position within this substring. As we have shown, the block $\Delta$ must contain the whole set $\Omega^{*}=\left\{1,2,3, \ldots, 2^{n-1}\right\}$. Hence, the group $G$ acts primitive on $\Omega^{\prime}$.

We proceed by shortly explaining the action of some specific permutations in $G$, which we will combine subsequently to yield the appropriate 3-cycle, forcing our group to be either alternating or symmetric.

(i) Structure of SHIFT_-1:

The general structure is as follows: SHIFT_-1 maps

$x \rightarrow x / 2, x$ even; $x \rightarrow(x-1) / 2+2^{n-1}, x$ odd.

Example $n=4:$ SHIFT_-1 $\equiv(0)(1,8,4,2)(3,9,12,6)(5,10)(7,11,13,14)(15)$

(ii) Structure of MUL_( $\left.2^{\mathrm{n}-1}+1\right)$ :

The general structure is as follows: MUL_ $\left(2^{n-1}+1\right)$ maps

$\mathrm{x} \rightarrow \mathrm{x}, \mathrm{x}$ even; $\mathrm{x} \rightarrow \mathrm{x}+2^{\mathrm{n}-1} \bmod 2^{\mathrm{n}}, \mathrm{x}$ odd;

Example $n=4:(0)(2)(4)(6)(8)(10)(12)(14)(1,9)(3,11)(5,13)(7,15)$

(iii) Structure of $A:=\left(\text { MUL }\left(2^{\mathrm{n}-1}+1\right)\right)^{\text {SHIFT }_{-}-1}$ :

Let $s$ be a cycle, $s=\left(s_{1}, s_{2}, s_{3}, \ldots, s_{k}\right)$, and let $t$ be an arbitrary permutation. Then tst $^{-1}=$ $\left(t\left(s_{1}\right), t\left(s_{2}\right), t\left(s_{3}\right), \ldots, t\left(s_{k}\right)\right)$. Hence we can see that, by the above analysis of the structure of MUL_ $\left(2^{n-1}+1\right)$ and SHIFT_-1, we get the following cycle structure of the permutation $\mathrm{A}:=\left(\mathrm{MUL} \_\left(2^{\mathrm{n}-1}+1\right)\right)^{\overline{\mathrm{SH} H \mathrm{~T}}-1}$ :

The fixed points of $A$ comprise of the set $\left\{x / 2,0 \leq x<2^{n}, x\right.$ even $\}=\left\{0,1,2,3, \ldots, 2^{n-1}-\right.$ 1). The transpositions in $A$ have the form $\left(x, x+2^{n-1}\right)^{\text {SHIFT-1 }}$, where $1 \leq x<2^{n-1}$, $x$ odd, which yields transpositions of the following form:

$\left((x-1) / 2+2^{n-1},\left(x+2^{n-1}-1\right) / 2+2^{n-1}\right)=\left((x-1) / 2+2^{n-1},(x-1) / 2+2^{n-1}+2^{n-2}\right)$, where 1 $\leq x<2^{n-1}$, $x$ odd. As we can see, the permutation $A$ does not move any element of size smaller than $2^{\mathrm{n}-1}$. Example $n=4: A=(8,12)(9,13)(10,14)(11,15)$.

(iv) Structure of $B:=A^{\mathrm{Mr}_{-}^{-1} \text { : }}$

First, notice that MUL -1 consists of two fixed points, namely 0 and $2^{n-1}$, together with transpositions of the form $\left(x, 2^{n}-x\right)$, for $x=1,2, \ldots, 2^{n-1}-1$.

Thus, under conjugation by MUL_-1, the fixed points of $\mathrm{A}$ move into the following set of fixed points of $B=A^{M\left(1 L_{-}-1\right.}:\{0\} \cup\left\{2^{n}-x, 1 \leq x \leq 2^{n-1}-1\right\}=\left\{0,2^{n-1}+1,2^{n-1}+2,2^{n-1}\right.$ $\left.+3, \ldots, 2^{n}-1\right\}$. Hence, the permutation $B$ does not move any element of size larger than $2^{\mathrm{n}-1}$. Example $\mathrm{n}=4: \mathrm{B}=(1,5)(2,6)(3,7)(4,8)$.

We combine the above observations and consider the product of $A$ and $B$. Both permutations, $A$ and $B$, are involutions. The only element which is going to be moved by $A$ as well as by $B$ is the element $2^{n-1}$. Therefore, the permutation $A B \in G$ consists of the union of those transpositions of $A$ and $B$ which do not contain the element $2^{n-1}$, together with exactly one further cycle of length 3 . This 3-cycle $\left(x, y, 2^{n-1}\right)$ stems from the product of a transposition $\left(x, 2^{n-1}\right)$ in $A$ with a transposition $\left(y, 2^{n-1}\right)$ in $B$. The square $C:=(A B)^{2} \in G$ is a 3 -cycle. Since the group $G$ is primitive, this proves that $G$ contains the alternating group of degree $2^{n}-1$. Further, the group $G$ contains odd permutations (take SHIFT_1, for example). Therefore, $G$ is isomorphic to the symmetric group of degree $2^{n}-1$. 


\subsection{Groups of Larger Subsets of Basic Operations}

In the above sections we studied the properties of each individual basic operation, as well as the combinatorial properties of different pairs of possible combinations of those basic operations. With this knowledge about specific groups, each generated by two types of generating functions, it is relatively easy to determine the algebraic structure of larger generating subsets. If, for example, we have a pair $\{\mathrm{A}, \mathrm{B}\}$ of basic operations, such that $\{A, B\}$ already generates the whole symmetric group of degree $2^{\mathrm{n}}$, then obviously every larger set $\{\mathrm{A}, \mathrm{B}, \mathrm{C}, \ldots\}$ of basic operations forms also a generating set for this group. This applies, for example, to the systems \{XOR, SHIFT, ADD\}, \{MUL, SHIFT, ADD $\},\{X O R$, SHIFT, MUL $\}$. As a funny property in this context, we can observe the following. Every addition $A D D \_c, 0 \leq c<2^{n}, n \in N$, can be executed as a sequence of multiplications modulo $2^{\mathrm{n}}$ and multiplications modulo $2^{\mathrm{n}}+1$, i.e. ADD $\subset$ $<$ MUL, MULT>. By this property we get additional results on subsets of basic operations which can generate the complete symmetric group of degree $2^{n}$. This holds, for example, for the generating set \{MULT, SHIFT, MUL\}.

\section{MIX-2 - A Simple System Generating the Symmetric Group}

\subsection{Description of MIX-2}

Based upon our analysis of basic encryption operations in the previous section, we will present a simple three-line algorithm named $M L X-2$ which is able to produce every possible permutation on its set of input vectors, i.e. MIX-2 can generate the symmetric group. MIX-2 uses only two different operations, namely SHIFT_a and ADD_b. Here, a and $b$ are odd. The register length $n$ depends on the computer; for example $n=64$. We will deduce that the round function of MIX-2, as well as the full-round MIX-2 scheme do generate the whole symmetric group of degree $2^{n}$.

Let $r$ denote the number of rounds, $M$ the input vector of length $n$, and let $\left(b_{1}, b_{2}, \ldots, b_{r}\right)$ be the first $r$ bits of the key K. Then the complete MIX-2 algorithm can be described as follows.

$$
\begin{aligned}
& \text { FOR }(\mathrm{i}=0 ; \mathrm{i}<\mathrm{r} ;++\mathrm{i}) \\
& \text { IF } \mathrm{b}_{\mathrm{i}} \quad \text { ADD_b(M); } \\
& \text { ELSE } \text { SHIFT_a(M); }
\end{aligned}
$$

As our key $\mathrm{K}$ we take a binary $\mathrm{k}$-bit vector $\mathrm{K}=\left(\mathrm{b}_{1}, \mathrm{~b}_{2}, \ldots, \mathrm{b}_{\mathrm{k}}\right) \mathrm{k}$ being (preferrably) a power of two, for example $k=256$. The number $r$ of rounds is equal to $k-\left(n+\log _{2}(n)\right.$ 2). If $\mathrm{n}=64$, for example, then we get $\mathrm{r}=256-64-6+2=188$. Here, the bit sequence $\left(b_{r+1}, b_{r+2}, \ldots, b_{r+n-1}, 1\right)$ provides the binary representation of the odd number $b$. Similiarly, the sequence $\left(b_{r^{+n}}, b_{r_{n+n}+1}, \ldots, b_{k}, 1\right)$ represents the odd number $a, 0 \leq a<2^{n}$. Now we can show the generator property of MIX-2. 
Theorem: The full-round MIX-2, as well as its set of round functions, can generate the symmetric group of degree $n$, where $n$ is the length of the input vectors.

Proof: As we have shown, the set of all SHIFT and ADD operations (SHIFT_x, ADD_y $\left.\mid 0 \leq x<n, 0 \leq y<2^{n}\right\}$ generates the symmetric group of degree $2^{n}$. But for every odd a and odd $\mathrm{b}$, we have $\langle$ SHIFT_a $>=\langle\{$ SHIFT_x $\mid 0 \leq \mathrm{x}<\mathrm{n}\}$ and $\langle$ ADD_b $>=\langle\{$ ADD_y $\mid$ $\left.0 \leq y<2^{n}\right\}>$. Hence, the two basic operations SHIFT_a and ADD_b, and therefore the round functions of MIX-2, do generate the symmetric group.

Now we have to study the resulting permutations of MIX-2 after $r$ rounds. Take key $\mathrm{K}_{1}$ $:=\left(1_{1}, 1_{2}, \ldots, 1_{r}, \ldots\right)$, then MIX-2 using this key corresponds with a simple ADD_rb operation. By the above definition it follows that $r$ is odd, hence $r b$ is odd and $\left\langle A D D \_r b>=\left\langle\left\{A D D \_y \mid 0 \leq y<2^{n}\right\}>\right.\right.$. Similiarly, by taking key $K_{0}=\left(0_{1}, 0_{2}, \ldots, 0_{r}\right.$, ...), we see that SHIFT_ra generates all SHIFT operations. Thus we have shown that MIX-2 generates the symmetric group.

\subsection{Poor Combinatorial Behaviour Through Different Functions in MIX-2}

In the above definition of MIX-2, we could have chosen other pairs of basic operations as well. Suppose we replace the addition modulo $2^{n}, A D D \_b$, by the similiar operation XOR $b$ (in this case, the constant $b$ does not have to be odd). The difference between the $\bar{X} O R \_b$ and the ADD_b operation consists, simply speaking, only in the consideration of carry bits. Some cryptographic properties of those carry bits have already been studied in another, non-combinatorial setting, by W. Meier and 0 . Staffelbach [Staf/Mei]. It is the propagation of those carry bits that saves the above algorithm from complete nonsense. The result of the replacement of ADD by XOR is disastrous. Independent from the actual number of rounds $r$, the resulting function

$$
\begin{aligned}
\text { FOR }(\mathrm{i}=0 ; \mathrm{i}<\mathrm{r} ;++\mathrm{i}) \\
\text { IF } \mathrm{b}_{\mathrm{i}} \quad \text { XOR_b(M); } \\
\text { ELSE SHIFT_a(M); }
\end{aligned}
$$

shrinks and is equivalent to a ,2-round version"

XOR_b'(M);

SHIFT_a'(M);

of the previous algorithm, where the corresponding key $K=\left(b_{1}, b_{2}, \ldots\right)$ can be chosen as $\mathrm{K}=(1,0, \ldots)$, for example.

Exactly the same problem occurs if, alternatively, we keep the ADD operator, but replace the SHIFT_a operator by MUL_a, i.e. multiplication modulo $2^{n}$. (Here we have to adjust key lengths appropriately, since the odd constant a now satisfies $0<\mathrm{a}<2^{\mathrm{n}}$.) Again, the complete algorithm

FOR $(\mathrm{i}=0 ; \mathrm{i}<\mathrm{r} ;++\mathrm{i})$

IF $b_{i}$ ADD_b(M);

ELSE MUL_a(M); 
is reducible to

ADD_b'(M);

MUL_a'(M);

since the affine general linear group $\operatorname{AGL}\left(1,2^{n}\right)$ generated by those operations is a semidirect product $A G L\left(1,2^{n}\right)=\mathrm{GH}$ of a group $\mathrm{G}$ by a group $\mathrm{H}$, where $\mathrm{G}$ is generated by the set $\left\{A D D \_x \mid 0 \leq x<2^{n}\right.$, $x$ odd $\}$ and $H$ is generated by the set $\{M U L y \mid 0 \leq y<$ $2^{\mathrm{n}}$, y odd $\}$. The shrinking property follows, similiarly as in the previous case of $<\mathrm{XOR}$, SHIFT $>$, from the fact that $\mathrm{GH}=\mathrm{HG}$.

Another weakness occurs if we consider the pairs $\{X O R, A D D\}$ or $\{X O R, M U L\}$ as ingredients to the above MIX-2 system. Both resulting permutation groups are imprimitive in the sense that the set of odd input vectors and the set of even input vectors (i.e. least significant bit equal to 1 or 0 , respectively) are permuted among themselves. Suppose we know one plaintext-ciphertext pair. Then, for example, if both text strings are even, we can deduce that every even (odd) plaintext will be mapped into an even (odd) ciphertext. For a cryptosystem, of course, this property would be intolerable. Note also that the multiplication operator MUL_a(.) always preserves the least significant bit of its operand. This fact, together with its relatively slow running time, is an additional disadvantage of the MUL operation.

Note that there exist examples in the literature, one of which had been designed by $\mathrm{S}$. Murphy, K. Paterson, and P. Wild [Mur/Pate/Wil], to demonstrate the existence of a weak cipher that nevertheless can generate the symmetric group. In their block encryption scheme, however, one of the bijections in the round function consists of a trivial permutation $\theta$ whose cycle decomposition is $\theta=(0)\left(2^{n-1}\right)\left(1,2, \ldots, 2^{n-1}-1,2^{n-1}+1\right.$, $\left.\ldots, 2^{n}-3,2^{n}-2,2^{n-1}\right)$. Thus, in all but a few cases, $\theta$ just increments its input by one, which is, of course, no very helpful encryption step. As the authors show, their cryptosystem, whose second encryption component is an XOR with the secret round key, can easily be broken by a known plaintext attack. We remark that MIX-2 is just a good example demonstrating a very simple algorithm having the potential to generate the whole symmetric group. We did not call it a cryptosystem and do not assume that MIX-2, without further ingredients and modifications, is of any use as a strong block cipher. Nevertheless, it is not that easy to break MIX-2, in comparison with its quite trivial structure. The above provides another reason to recommend the implementation of subroutines having strong combinatorial properties.

\section{Conclusions}

As we have shown, care has to be taken in the right combination of basic computer instructions to strengthen the combinatorial properties of an encryption algorithm. Since most schemes involve data-dependence of their basic encryption operations, some randomness assumptions have to be accepted in practice and further research in this direction should be undertaken to facilitate the determination of the group generated by 
a given block encryption scheme. We analyzed the algebraic structure of different combinations of some basic encryption operations from a purely combinatorial point of view. Different subsets of such operations yield an interesting variety of different permutation groups, e.g. semidirect products, affine linear groups, wreath products, and symmetric groups. Even though the above results are useful in theory at first, their cryptographic applications can be found in providing practical tools for the analysis of the algebraic structure of block encryption schemes and combinatorial evaluation of their subroutines.

\section{Bibliography}

[Came] P. J. Cameron, Finite permutation groups and finite simple groups, Bull. London Math. Soc. 13 (1981), pages 1-22.

[CampK/Wien] K. Campbell, and M. Wiener, DES is not a group, Proc. of Crypto'92, pages $512-520$.

[Cop/Gro] D. Coppersmith, and E. Grossman, Generators for certain alternating groups with applications to cryptography, SIAM Journal Appl. Math., 29(4), pages 624$627,1975$.

[Eve/Gol2] S. Even, and O. Goldreich, DES-like functions can generate the alternating group, IEEE Transaction on Inf. Theory, IT-29(6), pages 863-865, 1983.

[Hup] B. Huppert, Endliche Gruppen 1, 2. Nachdruck der 1.Auflage, Springer-Verlag Berlin, Heidelberg, New York 1967.

[Hup/Bla] B. Huppert, and N. Blackburn, Finite groups 3, Springer-Verlag Berlin, Heidelberg, New York 1982.

[Isa/Zie] I. M. Isaacs, and T. E. Zieschang, Generating symmetric groups, American Math. Mon., Oct. 1995, pages 734-739.

[Mur/Pate/Wil] S. Murphy, K. Paterson, and P. Wild, A weak cipher that generates the symmetric group, J.Cryptology (1994) 7, pages $61-65$.

[Pie/Zha] J. Pieprzyk, and X. Zhang, Permutation generators of alternating groups, Auscrypt'90, pages 237-244.

[Staf/Mei] O. Staffelbach, and W. Meier, Cryptographic Significance of the Carry for Ciphers Based on Integer Addition, Crypto'90, pages $601-614$.

[Wer] R. Wernsdorf, The l-round functions of DES generate the alternating group, Proc. of Eurocrypt' 92 , pages $99-112$

[Wiel] H. Wielandt, Finite permutation groups, Academic 1964. 